\title{
Heart failure in adults with congenital heart disease: a narrative review
}

\author{
Elvin Zengin ${ }^{1,2 \#}$, Christoph Sinning ${ }^{1,2,3 \#}$, Christopher Blaum ${ }^{1}$, Stefan Blankenberg ${ }^{1,3}$, Carsten Rickers ${ }^{2}$, \\ Yskert von Kodolitsch ${ }^{1}$, Paulus Kirchhof ${ }^{1,3,4}$, Nigel E. Drury ${ }^{4,5 *}$, Victoria M. Stoll ${ }^{4,5 *}$ \\ ${ }^{1}$ Department of Cardiology, University Heart \& Vascular Center Hamburg, Hamburg, Germany; ${ }^{2}$ Adult Congenital Heart Disease Section, \\ University Heart Center Hamburg, Hamburg, Germany; ${ }^{3}$ German Center for Cardiovascular Science (DZHK) Hamburg/Lübeck/Kiel, Hamburg, \\ Germany; ${ }^{4}$ Institute of Cardiovascular Sciences, University of Birmingham, Birmingham, UK; ${ }^{5}$ Department of Adult Congenital Cardiology \& \\ Cardiac Surgery, Queen Elizabeth Hospital Birmingham, Birmingham, UK \\ Contributions: (I) Conception and design: E Zengin, C Sinning, NE Drury, VM Stoll; (II) Administrative support: S Blankenberg, P Kirchhof, Y von \\ Kodolitsch; (III) Provision of study materials or patients: None; (IV) Collection and assembly of data: C Sinning, E Zengin; (V) Data analysis and \\ interpretation: E Zengin, C Sinning, NE Drury, VM Stoll; (VI) Manuscript writing: All authors; (VII) Final approval of manuscript: All authors. \\ \#These authors contributed equally to this work. \\ *These authors contributed equally for the senior authorship. \\ Correspondence to: Mrs. Dr. Elvin Zengin, MD. Department of Cardiology, University Heart- \& Vascular Center Hamburg, Hamburg, Germany. \\ Email: e.zengin@uke.de.
}

\begin{abstract}
The number of adults with congenital heart disease (ACHD) has increased over the last decades due to advancements in medical care, including interventional and surgical therapies. We are therefore more frequently challenged by the long-term consequences of palliative or corrective surgery carried out during childhood. Although patients with ACHD may develop conditions related to general cardiovascular risk factors, such as coronary artery disease, the most common complications leading to morbidity and mortality are arrhythmias, heart failure and thromboembolic events. For the management of arrhythmias, current recommendations regarding ablation and device therapy must be considered, whilst also taking into account the anatomical limitations of their congenital heart defect or surgical pathways. Heart failure treatment in acute and chronic settings must also consider the particular anatomy present, including the nature of the systemic ventricle. Treatments strategies for ACHD are typically extrapolated from the respective guidelines in non-ACHD patients, despite a lack of evidence to support this strategy. Right heart failure can be especially challenging to manage in conditions where either a systemic right ventricle or shunt lesions resulting in volume and/or pressure loading of the right ventricle are present. All physicians and cardiologists in particular should be acquainted with the most common diseases in ACHD, their complications and management regime, especially with regards to heart failure as this is a common reason for acute presentation in the emergency department.
\end{abstract}

Keywords: Congenital heart disease; heart failure; management; systemic ventricle

Submitted Jul 10, 2020. Accepted for publication Oct 27, 2020.

doi: $10.21037 / \mathrm{cdt}-20-632$

View this article at: http://dx.doi.org/10.21037/cdt-20-632

^ ORCID: 0000-0002-7331-8840. 


\section{Introduction}

The number of patients with adults with congenital heart disease (ACHD) is constantly increasing due to advances in the medical and surgical treatment options improving the outcome of children with complex congenital heart disease (1-4) (Figure 1 regarding epidemiology of ACHD patients). Current guidelines and other evidence suggest that treatment should be performed by trained physicians $(1,2,4)$ with appropriate infrastructure for the specialised care for these patients (5-7).

The number of patients with ACHD has increased by about $50 \%$ compared with the number of children with congenital heart defects since the beginning of the new millennium (8-11). Currently adult patients account for about two thirds of all patients with congenital heart disease (12) and this change in demographics is resulting in a mortality shift from infancy to adulthood $(5,13)$. Patients with complex cardiac disease after palliative or corrective surgery can present with the surgical sequelae of heart defects $(2,4-6,14-16)$. The most common defects resulting in emergency hospitalisation of the patient are the univentricular heart, followed by tetralogy of Fallot, common arterial trunk, and discordant connections such as transposition of the great arteries following Senning or Mustard operations and congenitally corrected transposition of the great arteries (5,6,14-16). Frequent reasons for admittance to the emergency department are arrhythmias, as well as heart failure (HF), thromboembolic complications resulting in stroke or pulmonary embolism and non-cardiac causes $(2,4-6,16)$.

This manuscript aims to describe the most common diseases in detail to elucidate diagnostic, treatment and management strategies for patients with ACHD.

\section{Objectives}

This review summarizes the aetiology, diagnostic process and treatment of heart failure in ACHD patients. In addition, the most recent guidelines were included, accomplishing the goal of a better treatment algorithm. Further a focus of the review is to give examples of special situations which are often encountered in ACHD patients presenting with heart failure like treatment with cardiac resynchronization therapy or potential treatment with a ventricular assist device. We present the following article in accordance with the NARRATIVE REVIEW reporting checklist (available at http://dx. doi. org/10. 21037/cdt-20-
632).

\section{Methods}

\section{Literature search}

A literature search was performed in PubMed covering publications up to April 2020. The following combinations of keywords were used: heart failure and CHD, morbidity and mortality in ACHD, guidelines heart failure and ACHD, guidelines diagnostic and treatment of patients with congenital heart disease. These search terms had to be identified anywhere in the text in the articles. The authors as well did choose literature depicting the current guidelines or multicenter trials, however most of the literature are of observational studies and guidelines for patients with ACHD have the evidence level of expert opinion.

Both qualitative and quantitative studies were considered to elucidate the use of the different aspects regarding congenital heart disease, anticoagulation and combination of both. The search was restricted to original research, humans, and papers published in English at any date. All abstracts were reviewed to assess whether the article met the inclusion criteria. After this selection process, a manual search of the reference lists of all eligible articles was performed. Two authors (i.e., EZ and CS) assessed independently the methodological quality of the qualitative and quantitative studies prior to their inclusion in the review.

\section{Epidemiology of heart failure (HF) in ACHD}

Patients with ACHD and complex cardiac anatomy are prone to develop HF over the course of their lives, especially those with a univentricular circulation. Signs of $\mathrm{HF}$ have been reported in about $22 \%$ of adults with transposition of the great arteries (TGA) following an atrial switch (Senning or Mustard operation), 32\% of adults with congenitally corrected transposition (ccTGA) and $40 \%$ of adults following Fontan completion $(17,18)$. In patients with $\mathrm{ACHD}, \mathrm{HF}$ is responsible for about $20 \%$ of mortality, often during early adulthood (19). Current clinical guidelines may not be directly applicable to this complex cohort of patients due to a lack of evidence $(18,20,21)$. For the treatment of patients with ACHD who develop HF, it is important to assess if there are any residual hemodynamic lesions such as a shunt or atrioventricular valve regurgitation $(22,23)$. 

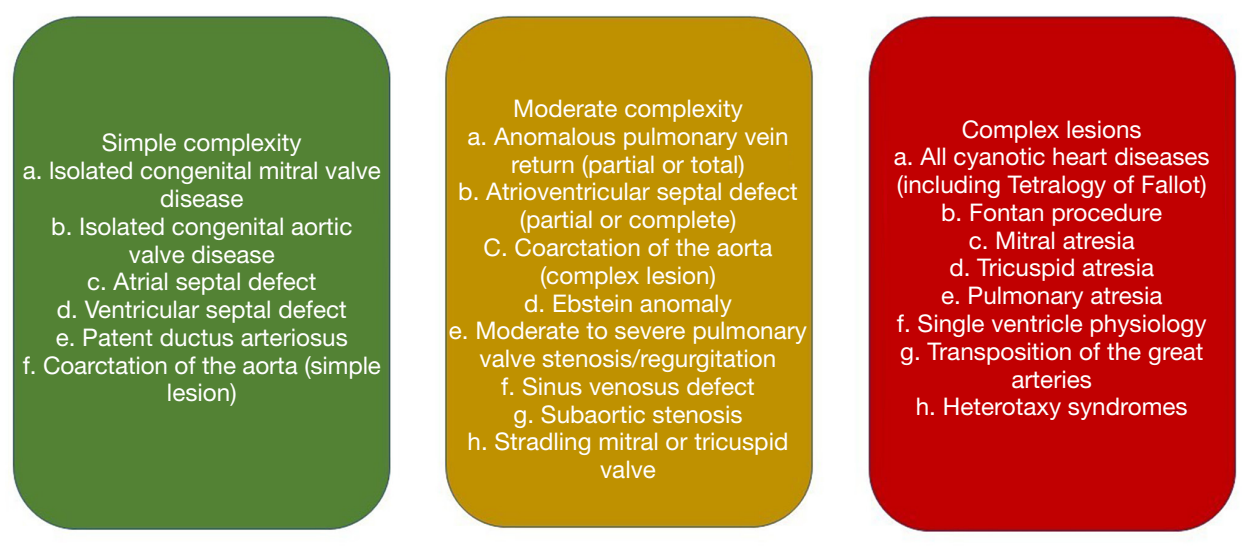

Increasing morbidity and mortality of the congenital heart defect

Figure 1 Complexity of congenital heart disease with prevalence of the disease. Prevalence of complex lesions is increasing due to a better survival in patients with moderate or complex congenital heart disease.

\section{Limitation of exercise capacity in patients with ACHD and heart failure}

Patients with ACHD often do not present with the same symptoms as other patients with $\operatorname{HF}(17,18,20,24)$ as they have already adapted to living with impaired physical exercise tolerance. It is therefore important to gain objective evidence from cardiopulmonary exercise testing to detect a decline in physical exercise tolerance in order to identify patients who may benefit from therapeutic interventions and those at risk of an adverse outcome $(25,26)$. Many patients with congenital heart defects will have impaired peak oxygen consumption, this is due to multiple factors including inadequate cardiac reserve, poor heart rate response to exercise, raised pulmonary pressures upon exercise, poor pre-conditioning, or even due to restrictions recommended by physicians.

\section{Comorbidities and anatomic variations predisposing to heart failure}

\section{Systemic right ventricle}

In patients with TGA following an atrial switch, hypoplastic left heart syndrome following the Norwood operation, and uncorrected ccTGA, the morphologic right ventricle is in the systemic circulation. The morphologic right ventricle is a thin-walled structure with only two muscle layers, compared with three layers in the morphologic left ventricle, and is heavily dependent on loading conditions, especially changes in afterload $(18,20,22,23)$; it is therefore unable to sustain the pressure generation required to pump blood around the systemic circulation and over time is predisposed to fail. Additionally for the development of heart failure the presence of a trileaflet tricuspid valve rather than a bileaflet mitral valve is significant. These patients frequently develop tricuspid regurgitation which exacerbates heart failure symptoms, especially in Ebsteinlike tricuspid dysplasia, which is the most common cardiac abnormality related to ccTGA $(22,23,27)$.

\section{Pulmonary vascular disease}

Evaluation of the pulmonary circulation is of paramount importance in patients presenting with subpulmonary RV failure and those with a Fontan circulation $(17,22,23)$. Pulmonary arterial hypertension $(\mathrm{PAH})$ is common in ACHD as a result of anatomic heterogeneity and requires careful characterisation to target specific medical treatments addressing $\mathrm{PAH}$, whilst misclassification can be deleterious for patients assessed for heart transplantation $(17,22)$. Most patients with ACHD and PAH have WHO (World Health Organization) group $1 \mathrm{PAH}$, with a pulmonary vascular resistance (PVR) of $\geq 3$ Wood units. Some may have pulmonary hypertension WHO class 2 with an increased pulmonary wedge pressure, whilst others, especially those with TGA or ccTGA, may have components of both pre- and postcapillary pulmonary hypertension which are most commonly related to WHO 
class 2 or $5(17,28)$. Postcapillary pulmonary hypertension can arise due to a failing systemic right ventricle in case of ccTGA or TGA after atrial switch procedure and precapillary pulmonary arterial hypertension can be associated with shunt lesions or abnormalities of the pulmonary vasculature $(4,17)$.

Meticulous assessment of the hemodynamic status is crucial to determine whether a patient would require from lung transplantation in addition to heart transplantation (17). In patients with ACHD, development of pulmonary hypertension is associated with a 2 -fold increase in all-cause mortality (17).

\section{Shunts, collaterals and baffles}

During the assessment of patients with complex CHD, it is important to consider the presence or persistence of shunts and collaterals as these may cause additional volume and/or pressure load. Pre-tricuspid shunt lesions, e.g., atrial septal defect, result in volume loading on the pulmonary circulation whilst post-tricuspid shunt lesions, e.g., ventricular septal defect and patent ductus arteriosus, cause volume loading of the left ventricle that may lead to a decline in left heart function. In a Fontan circulation, a spontaneous or surgically created fenestration may cause right-to-left shunting with arterial desaturation (22). Patients with TGA after atrial switch procedure show an impaired capacitance and conduit function of the baffles (29) and so a fast heart rate, such as during atrial fibrillation with a fast ventricular response, is not well tolerated, with reduced diastolic ventricular filling causing decreased cardiac output, that may even lead to cardiogenic shock $(22,29)$. The baffles in patients with Fontan circulation are prone to narrowing or developing stenosis resulting in filling disorders of the single ventricle (4).

\section{Heart failure management in ACHD and specific considerations}

The number of patients with ACHD presenting to the emergency department with heart failure symptoms increases with the age and survival of the patients $(9-11,18)$. Concerning the treatment strategy it has implications whether the anatomy of the patients is related to a morphologic left or right ventricle in the systemic circulation, whether the sub-pulmonary right ventricle is impaired, or whether the patient has a Fontan circulation
$(18,30,31)$. HF in patients with ACHD is often complicated by comorbidities resulting from the initial palliative or corrective surgical procedure. Frequently impaired renal function or haematological disorders with increased haematocrit due to chronic systemic cyanosis can be observed. In patients with a Fontan circulation, chronically elevated pressure in the systemic venous pathways and hepatic veins leads to hepatic enlargement, progressive fibrosis and even cirrhosis and in some cases hepatocellular carcinoma (18).

\section{Diagnosis of HF in patients with ACHD}

Knowledge of the underlying congenital heart defect as well as the history of previous surgeries and interventions is crucial for the diagnosis of HF in ACHD. The change in symptoms might be subtle, a common first symptom being the onset of arrhythmia (18). Besides the need for a regular work-up with ECG and imaging including advanced imaging modalities like cardiac magnetic resonance imaging or computer tomography, two cornerstones for diagnosis are laboratory testing including $\mathrm{N}$-terminal pro-B-type natriuretic peptide (NT-proBNP) and cardiopulmonary exercise testing (CPET). Two challenges for diagnosis are the symptomatic patients with normal systemic ventricular function and the asymptomatic patient with impaired function of the systemic right ventricle. In these two constellations a decrease of peak oxygen consumption by $25 \%$ in CPET or two-fold increase of NT-proBNP during 6 months of follow-up are indicators to start treatment (18).

An important difference to other current HF guidelines is the threshold of the ejection fraction being $<40 \%$ before medical treatment is recommended (18). ACHD patients with heart failure and an anatomical left ventricle as systemic ventricle with a reduced ejection fraction $<40 \%$ are treated with the same medication as recommended in non-ACHD patients (18). Most commonly reninangiotensin-aldosterone-system blockers are used in addition to beta-blockers, mineralocorticoid receptor antagonists and diuretics or digoxin. If the failing ventricle is an anatomic right ventricle the medication is only used in symptomatic patients (18). Patients with a single ventricle are handled the same way. These patients are treated with the suggested medication if the ventricle is an anatomic left ventricle irrespective of symptoms and in case of an anatomic right single ventricle only if the patient is symptomatic (18). 


\section{Treatment of HF in ACHD}

Patients with impaired function of the systemic left ventricle are often treated according to the ESC guidelines on HF $(18,21)$. Most commonly the treatment includes therapy with an ACE-inhibitor, AT-1 receptor blocker or betablocker, as well as diuretics for symptomatic relief.

In patients with a systemic morphologic right ventricle, HF is likely to develop over time since the anatomy and geometry of the right ventricle are not adapted to generate the needed perfusion pressure for the systemic circulation $(18,30)$. Whilst clear evidence for recommendations is lacking, asymptomatic patients with a failing systemic right ventricle are often treated with beta-blockers. However, symptomatic patients are treated analogous to patients with a failing morphologic left ventricle (18). If the sub-pulmonary morphologic left or right ventricle is compromised, no treatment is recommended in asymptomatic patients, but in the presence of symptoms, a combination of diuretics can provide symptomatic relief (18).

For patients with a functionally univentricular heart who have been palliated with a Fontan circulation, the presence of HF symptoms in conjunction with impaired ventricular function indicates the need for treatment and symptom relief, with no difference in approach to those with a systemic morphologic left or right ventricle (18). The largest benefits have been reported from reducing pulmonary vascular resistance with specific therapy such as phosphodiesterase inhibitors, or reducing afterload with beta blockers, whilst diuretics are only recommended if fluid overload is present (18).

\section{Treatment of acute heart failure in patients with ACHD}

Currently there are no specific guidelines for the treatment of patients with ACHD and acute heart failure (AHF), hence treatment recommendations are based on those for the general heart failure population, with a specific focus on comorbidities or anatomic variations present. Patients with ACHD and advanced HF may also be considered for ECMO therapy or ventricular assist device therapy as a bridge to destination therapy, if available and appropriate (18,21-23).

Heart failure therapy in patients with ACHD needs to take into account the individual anatomic circumstances and frequent comorbidities complicating therapy for an individual patient. Optimal outcomes are achieved when these patients are managed by specialist ACHD teams
$(17,24)$.

\section{Device treatment with intra-cardiac cardioverter defibrillator (ICD) in patients with ACHD and beart failure}

Evidence for the use of an ICD in patients with ACHD is limited and is frequently based on expert opinion as the most commonly derived data on ICD use in ACHD is from observational studies (4,32-34). In general, the indications as outlined in the current ESC guidelines regarding ICD indications for secondary prevention can be extrapolated to ACHD (32-34). Patients with repaired tetralogy of Fallot, TGA following atrial switch, and uncorrected ccTGA are reported to have a high incidence of ventricular arrhythmias and are often candidates for ICD implantation in the context of secondary prevention (32-34). Regarding primary prevention, guidelines concur that patients with ACHD fulfilling the current ICD criteria with a left ventricular ejection fraction $<35 \%$, biventricular physiology, optimally treated heart failure, and NYHA symptom class II or III, are candidates for ICD implantation (32-34). However, appropriate selection of candidates remains challenging, weighing the benefits against the potential risks of infection or venous obstruction due to intravascular lead placement (32-34) as well as the potential technical challenges posed in patients with complex anatomy.

\section{Cardiac resynchronization therapy (CRT) in patients with ACHD}

The efficacy of CRT varies in patients with ACHD according to the underlying disease and anatomic substrate and whether a systemic morphologic left, right or functionally univentricular heart is present $(32,34)$. Whilst reported data in ACHD is mostly derived from observational studies, CRT has a class I indication in patients with a systemic morphologic left ventricle and who fulfil the same indication criteria for CRT as non-ACHD patients $(34,35)$. However, even if the systemic ventricle is a morphologic right ventricle, CRT can be considered if the ejection fraction is $<35 \%$, the ventricle is dilated, the patient displays NYHA class II to IV (ambulatory) symptoms, and a complete right bundle branch block with a QRS complex $\geq 150 \mathrm{msec}$ is present $(34,35)$. An additional important potential indication for CRT in patients with ACHD is the presence of a pacing threshold $>40 \%$ during exchange of a pre-existing device or if a high pacing threshold is 
anticipated (34,35). Reports of patients with ACHD who are potential candidates for heart transplantation improving their functional status after CRT implantation to the extent that they can be removed from the transplant waiting list, suggests that CRT indications should be reviewed in the ACHD population $(34,35)$.

\section{Ventricular assist devices: special considerations in patients with ACHD}

Considering the rising number of patients with ACHD and the increase in HF in this population, there is a need to evaluate additional treatment options. Among the novel therapies developed for HF, durable mechanical circulatory support in the form of ventricular assist devices (VADs) can function as a bridge to transplantation. These devices have been shown to decrease mortality in end-stage HF among non-transplant candidates and appear to offer a similar benefit to transplant candidates who are deteriorating whilst awaiting transplantation $(21,36)$. For VAD implantation, patients should be considered according to whether they have a systemic morphologic left or right ventricle or a functionally univentricular heart. In the largest study to date, more patients with ACHD required biventricular ventricular assist or a total artificial heart than non-ACHD patients, and they more often had concomitant dysfunction of the subpulmonary ventricle (either morphologic left or right ventricle) and pulmonary hypertension than their non-ACHD counterparts (36). Patients with ACHD had higher rates of mortality and adverse events than nonACHD patients in the study, but the increased mortality rate was attributable solely to the higher use of biventricular ventricular assist devices and total artificial hearts. Conversely, the two groups who had VAD implantation to support the systemic ventricle alone, demonstrated similar survival regardless of cardiac anatomy (36).

\section{Heart transplantation in patients with ACHD}

In advanced HF, listing for heart transplantation is the ultimate option for disease management (21). However, patients with ACHD frequently have numerous adverse features including pulmonary arterial hypertension and other advanced organ involvement, such as liver disease in patients with Fontan circulation, resulting in a high waiting list mortality $(17,37)$. Patients removed from the waiting list have an especially poor prognosis as they cannot be bridged to transplantation with a VAD or have severe HF with multi-organ failure most commonly involving the liver and/ or kidneys $(37,38)$. During transplant assessment in patients with ACHD, a comprehensive anatomic and hemodynamic assessment is warranted to identify potentially correctable residual lesions from the original congenital heart defect and subsequent surgical or catheter procedures (17). Patients with moderate or complex congenital heart disease are closely screened and potential candidates for transplantation can be identified early, before multi-organ involvement prohibits potential listing. Until the definition of clear guidelines for the management of these patients, they should be screened especially carefully at a centre familiar with transplantation in congenital heart disease $(4,18)$.

\section{Summary}

Heart failure is one of the most common causes of death in the increasing number of patients with ACHD. These patients frequently have impaired function of the cardiovascular system, but often do not present early because they have adapted to living with impaired function from childhood. Thus rigorous follow-up of patients with ACHD is necessary, especially for those who have had palliative surgery or with underlying complex lesions. Current guidelines support the use of imaging, cardiopulmonary exercise testing and biomarkers as key investigations in disease progression monitoring. Treatment is based on the extrapolation of current knowledge from acquired heart disease in patients with systemic left ventricular dysfunction, even though the stabilising or beneficial effect of these medications is yet to be demonstrated in patients with ACHD. In advanced heart failure, hemodynamic assessment is essential to identify patients who may be candidates for heart transplantation. In those who are not suitable for listing for transplantation or with precluding comorbidities, a durable ventricular assist device should be considered whilst taking into account that only patients with singular VAD for the systemic ventricle had an outcome comparable to non-ACHD patients. Whilst heart failure is frequently encountered in patients with ACHD and several treatment options are available, it is essential that they are managed by a team specialising in ACHD.

\section{Acknowledgments}

Funding: None. 


\section{Footnote}

Provenance and Peer Review: This article was commissioned by the editorial office, Cardiovascular Diagnosis and Therapy for the series "Current Management Aspects in Adult Congenital Heart Disease (ACHD): Part III". The article has undergone external peer review.

Reporting Checklist: The authors have completed the NARRATIVE REVIEW reporting checklist. Available at http://dx. doi. org/10. 21037/cdt-20-632

Peer Review File: Available at http://dx. doi. org/10. 21037/ cdt $-20-632$

Conflicts of Interest: All authors have completed the ICMJE uniform disclosure form (available at http:// dx. doi. org/10. 21037/cdt-20-632). The series "Current Management Aspects in Adult Congenital Heart Disease (ACHD): Part III" was commissioned by the editorial office without any funding or sponsorship. YVK served as the unpaid Guest Editor of the series and an unpaid editorial board member of Cardiovascular Diagnosis and Therapy from Feb 2018 to Jan 2020. Dr. Blankenberg reports grants and personal fees from Abbott Diagnostics, grants and personal fees from Bayer, grants from SIEMENS, grants from Singulex, grants and personal fees from Thermo Fisher, personal fees from Abott, personal fees from Astra Zeneca, personal fees from AMGEN, personal fees from Medtronic, personal fees from Pfizer, personal fees from Roche, personal fees from Novartis, personal fees from Siemens Diagnostics, outside the submitted work. Dr. Kirchhof reports non-financial support from European Union, nonfinancial support from British Heart Foundation, nonfinancial support from Leducq Foundation, non-financial support from Medical Research Council (UK), nonfinancial support from German Centre for Cardiovascular Research, outside the submitted work; In addition, Dr. Kirchhof has a patent WO 2015140571 issued, and a patent WO 2016012783 issued and research support for basic, translational, and clinical research projects from several drug and device companies active in atrial fibrillation, and has received honoraria from several such companies in the past, but not in the last three years. The authors have no other conflicts of interest to declare.

Ethical Statement: The authors are accountable for all aspects of the work in ensuring that questions related to the accuracy or integrity of any part of the work are appropriately investigated and resolved.

Open Access Statement: This is an Open Access article distributed in accordance with the Creative Commons Attribution-NonCommercial-NoDerivs 4.0 International License (CC BY-NC-ND 4.0), which permits the noncommercial replication and distribution of the article with the strict proviso that no changes or edits are made and the original work is properly cited (including links to both the formal publication through the relevant DOI and the license). See: https://creativecommons.org/licenses/by-nc-nd/4.0/.

\section{References}

1. Cordina R, Nasir Ahmad S, Kotchetkova I, et al. Management errors in adults with congenital heart disease: prevalence, sources, and consequences. Eur Heart J 2018;39:982-9.

2. Warnes CA, Williams RG, Bashore TM, et al. ACC/ AHA 2008 Guidelines for the Management of Adults with Congenital Heart Disease: a report of the American College of Cardiology/American Heart Association Task Force on Practice Guidelines (writing committee to develop guidelines on the management of adults with congenital heart disease). Circulation 2008;118:e714-833.

3. Deanfield J, Thaulow E, Warnes C, et al. Management of grown up congenital heart disease. Eur Heart J 2003;24:1035-84.

4. Baumgartner H, De Backer J, Babu-Narayan SV, et al. 2020 ESC Guidelines for the management of adult congenital heart disease. Eur Heart J 2021;42:563-645.

5. Kaemmerer H, Bauer U, Pensl U, et al. Management of emergencies in adults with congenital cardiac disease. Am J Cardiol 2008;101:521-5.

6. Kaemmerer H, Fratz S, Bauer U, et al. Emergency hospital admissions and three-year survival of adults with and without cardiovascular surgery for congenital cardiac disease. J Thorac Cardiovasc Surg 2003;126:1048-52.

7. Tutarel $\mathrm{O}$. Acquired heart conditions in adults with congenital heart disease: a growing problem. Heart 2014;100:1317-21.

8. Marelli AJ, Mackie AS, Ionescu-Ittu R, et al. Congenital heart disease in the general population: changing prevalence and age distribution. Circulation 2007;115:163-72.

9. Singh S, Desai R, Fong HK, et al. Extra-cardiac comorbidities or complications in adults with congenital 
heart disease: a nationwide inpatient experience in the United States. Cardiovasc Diagn Ther 2018;8:814-9.

10. Neidenbach RC, Lummert E, Vigl M, et al. Non-cardiac comorbidities in adults with inherited and congenital heart disease: report from a single center experience of more than 800 consecutive patients. Cardiovasc Diagn Ther 2018;8:423-31.

11. Neidenbach R, Niwa K, Oto O, et al. Improving medical care and prevention in adults with congenital heart diseasereflections on a global problem-part I: development of congenital cardiology, epidemiology, clinical aspects, heart failure, cardiac arrhythmia. Cardiovasc Diagn Ther 2018;8:705-15.

12. Marelli AJ, Ionescu-Ittu R, Mackie AS, et al. Lifetime prevalence of congenital heart disease in the general population from 2000 to 2010. Circulation 2014;130:749-56.

13. Khairy P, Ionescu-Ittu R, Mackie AS, et al. Changing mortality in congenital heart disease. J Am Coll Cardiol 2010;56:1149-57.

14. Koh AS, Yap BT, Le Tan J. Emergency admissions in Asians with adult congenital heart disease. Int J Cardiol 2011;151:54-7.

15. Lin YS, Liu PH, Wu LS, et al. Major adverse cardiovascular events in adult congenital heart disease: a population-based follow-up study from Taiwan. BMC Cardiovasc Disord 2014;14:38.

16. Bédard E, Shore DF, Gatzoulis MA. Adult congenital heart disease: a 2008 overview. Br Med Bull 2008;85:151-80.

17. Menachem JN, Schlendorf KH, Mazurek JA, et al. Advanced Heart Failure in Adults With Congenital Heart Disease. JACC Heart Fail 2020;8:87-99.

18. Budts W, Roos-Hesselink J, Radle-Hurst T, et al. Treatment of heart failure in adult congenital heart disease: a position paper of the Working Group of GrownUp Congenital Heart Disease and the Heart Failure Association of the European Society of Cardiology. Eur Heart J 2016;37:1419-27.

19. Verheugt CL, Uiterwaal CS, van der Velde ET, et al. Mortality in adult congenital heart disease. Eur Heart J 2010;31:1220-9.

20. Stout KK, Broberg CS, Book WM, Cecchin F, Chen JM, Dimopoulos K, et al. Chronic Heart Failure in Congenital Heart Disease: A Scientific Statement From the American Heart Association. Circulation 2016;133:770-801.

21. Ponikowski P, Voors AA, Anker SD, et al. 2016 ESC Guidelines for the diagnosis and treatment of acute and chronic heart failure: The Task Force for the diagnosis and treatment of acute and chronic heart failure of the European Society of Cardiology (ESC)Developed with the special contribution of the Heart Failure Association (HFA) of the ESC. Eur Heart J 2016;37:2129-200.

22. Van De Bruaene A, Meier L, Droogne W, et al. Management of acute heart failure in adult patients with congenital heart disease. Heart Fail Rev 2018;23:1-14.

23. Van De Bruaene A, Hickey EJ, Kovacs AH, et al. Phenotype, management and predictors of outcome in a large cohort of adult congenital heart disease patients with heart failure. Int J Cardiol 2018;252:80-7.

24. Negishi J, Ohuchi H, Miyazaki A, et al. Clinical Characteristics of Adult Patients With Congenital Heart Disease Hospitalized for Acute Heart Failure. Circ J 2018;82:840-6.

25. Kempny A, Dimopoulos K, Uebing A, et al. Reference values for exercise limitations among adults with congenital heart disease. Relation to activities of daily life-single centre experience and review of published data. Eur Heart J 2012;33:1386-96.

26. Diller GP, Dimopoulos K, Okonko D, et al. Exercise intolerance in adult congenital heart disease: comparative severity, correlates, and prognostic implication. Circulation 2005;112:828-35.

27. Graham TP Jr, Bernard YD, Mellen BG, et al. Longterm outcome in congenitally corrected transposition of the great arteries: a multi-institutional study. J Am Coll Cardiol 2000;36:255-61.

28. Brida M, Diller GP, Gatzoulis MA. Systemic Right Ventricle in Adults With Congenital Heart Disease: Anatomic and Phenotypic Spectrum and Current Approach to Management. Circulation 2018;137:508-18.

29. Derrick GP, Narang I, White PA, et al. Failure of stroke volume augmentation during exercise and dobutamine stress is unrelated to load-independent indexes of right ventricular performance after the Mustard operation. Circulation 2000;102:III154-9.

30. Konstam MA, Kiernan MS, Bernstein D, et al. Evaluation and Management of Right-Sided Heart Failure: A Scientific Statement From the American Heart Association. Circulation 2018;137:e578-e622.

31. Krieger EV, Valente AM. Heart failure treatment in adults with congenital heart disease: where do we stand in 2014? Heart 2014;100:1329-34.

32. Hernández-Madrid A, Paul T, Abrams D, et al. Arrhythmias in congenital heart disease: a position paper of the European Heart Rhythm Association (EHRA), Association for European Paediatric and Congenital 
Cardiology (AEPC), and the European Society of Cardiology (ESC) Working Group on Grown-up Congenital heart disease, endorsed by HRS, PACES, APHRS, and SOLAECE. Europace 2018;20:1719-53.

33. Priori SG, Blomstrom-Lundqvist C, Mazzanti A, et al. 2015 ESC Guidelines for the management of patients with ventricular arrhythmias and the prevention of sudden cardiac death: The Task Force for the Management of Patients with Ventricular Arrhythmias and the Prevention of Sudden Cardiac Death of the European Society of Cardiology (ESC). Endorsed by: Association for European Paediatric and Congenital Cardiology (AEPC). Eur Heart J 2015;36:2793-867.

34. Khairy P, Van Hare GF, Balaji S, et al. PACES/HRS Expert Consensus Statement on the Recognition and Management of Arrhythmias in Adult Congenital Heart Disease: developed in partnership between the Pediatric and Congenital Electrophysiology Society (PACES) and the Heart Rhythm Society (HRS). Endorsed by the governing bodies of PACES, HRS, the American College of Cardiology (ACC), the American Heart Association (AHA), the European Heart Rhythm Association (EHRA), the Canadian Heart Rhythm Society (CHRS), and the
International Society for Adult Congenital Heart Disease (ISACHD). Heart Rhythm 2014;11:e102-65.

35. Brignole M, Auricchio A, Baron-Esquivias G, et al. 2013 ESC Guidelines on cardiac pacing and cardiac resynchronization therapy: the Task Force on cardiac pacing and resynchronization therapy of the European Society of Cardiology (ESC). Developed in collaboration with the European Heart Rhythm Association (EHRA). Eur Heart J 2013;34:2281-329.

36. VanderPluym CJ, Cedars A, Eghtesady P, et al. Outcomes following implantation of mechanical circulatory support in adults with congenital heart disease: An analysis of the Interagency Registry for Mechanically Assisted Circulatory Support (INTERMACS). J Heart Lung Transplant 2018;37:89-99.

37. Alshawabkeh LI, Hu N, Carter KD, et al. Wait-List Outcomes for Adults With Congenital Heart Disease Listed for Heart Transplantation in the U.S. J Am Coll Cardiol 2016;68:908-17.

38. Crossland DS, Jansen K, Parry G, et al. Outcome following heart transplant assessment in adults with congenital heart disease. Heart 2019;105:1741-7.

Cite this article as: Zengin E, Sinning C, Blaum C, Blankenberg S, Rickers C, von Kodolitsch Y, Kirchhof P, Drury NE, Stoll VM. Heart failure in adults with congenital heart disease: a narrative review. Cardiovasc Diagn Ther 2021;11(2):529-537. doi: 10.21037/cdt-20-632 